\title{
Temperature Dependence of the LabPET Small-Animal PET Scanner
}

\author{
Vincent Keereman, Christian Vanhove, Roel Van Holen, Stefaan Vandenberghe
}

\begin{abstract}
INTRODUCTION In quantitative PET imaging it is important to correct for all image-degrading effects, for example detector efficiency variation. Detector efficiency variation depends on the stability of detector efficiency when operating conditions vary within normal limits. As the efficiency of APDbased light detection strongly depends on ambient temperature, temperature-dependent detector efficiency normalization may be needed in APD-based PET scanners. We have investigated the temperature dependence of the LabPET APD-based small-animal PET scanner.
\end{abstract}

MATERIALS AND METHODS First a simulation study was performed to evaluate the effect of different APD temperature coefficients on the temperature dependence of scanner sensitivity. Five experiments were also performed. First the immediate effect of temperature changes on scanner sensitivity was evaluated. Second, the effect of temperature changes that have stabilized for a few hours was investigated. In a third experiment the axial sensitivity profile was acquired at $21^{\circ} \mathrm{C}$ and $24^{\circ} \mathrm{C}$. Next, two acquisitions of the NEMA image quality phantom (at $21^{\circ} \mathrm{C}$ and $23^{\circ} \mathrm{C}$ ) were performed and absolute quantification was done based on normalization scans acquired at the correct and incorrect temperature. Finally, the feasibility of maintaining a constant room temperature and the stability of the scanner sensitivity under constant room temperature was evaluated.

RESULTS Simulations showed that the relation between temperature-dependent APD gain changes and scanner sensitivity is quite complex. A temperature deviation leading to a 1\% change in APD gain corresponds to a much larger change in scanner sensitivity due to the shape of the energy histogram. In the first and second experiment a strong correlation between temperature and scanner sensitivity was observed. Changes of $2.24 \mathrm{kcps} / \mathrm{MBq}$ and $1.64 \mathrm{kcps} / \mathrm{MBq}$ per ${ }^{\circ} \mathrm{C}$ were seen for immediate and stabilized temperature changes respectively. The NEMA axial sensitivity profile also showed a decrease in sensitivity at higher temperature. The quantification experiment showed that a larger quantification error (up to $13 \%$ ) results when a normalization scan acquired at the incorrect temperature is used. In the last experiment, temperature variability was $0.19^{\circ} \mathrm{C}$ and counts varied by 10.2 Mcts $(1.33 \%)$.

CONCLUSION The sensitivity of the LabPET small-animal PET scanner strongly depends on room temperature. Therefore, room temperature should be kept as stable as possible and temperature-dependent detector efficiency normalization should be used. However, with constant room temperature excellent scanner stability is observed. Temperature should be kept constant within $0.5^{\circ} \mathrm{C}$ and weekly normalization scans are recommended. tion.

Index Terms-PET, CT, MRI, PET-MRI, attenuation correc-

\section{INTRODUCTION}

V. Keereman, C. Vanhove, R. Van Holen and S. Vandenberghe are with Department of Electronics and Information Systems, MEDISIP, Ghent University-IBBT-IBiTech, De Pintelaan 185 Block B, 9000 Gent, Belgium (email: Vincent.Keereman@ugent.be)
$\mathbf{I}$ $\mathrm{N}$ positron emission tomography (PET) different effects degrade image quality, such as photon attenuation and scatter, geometric sensitivity and detector efficiency variation. Accurate correction of these effects is necessary to obtain quantitative PET images. Correction methods are available for all of these effects. In particular, detector efficiency normalization is straightforward if the efficiency of all detectors in the system at the time of acquisition is known[1]. Detector efficiency is usually determined from a normalization scan, performed e.g. with a rotating line source, plane source or a uniform phantom. These normalization scans are often timeconsuming. Therefore one would preferably only perform normalization scans when necessary, i.e. when detector efficiency changes significantly. This depends on the stability of detector efficiency when operating conditions vary within normal limits.

Avalanche photodiodes are nowadays being routinely used for the detection of light emitted by scintillation crystals in PET scanners. Due to their small size they are of interest in small animal PET scanners and as they are insensitive to magnetic fields (contrary to photomultiplier tubes) they can be used in integrated PET-MR scanners[2]. It is well known that the gain of avalanche photodiodes (APDs) strongly depends on ambient temperature[3]. Therefore, the detector efficiency of the detectors of an APD-based PET scanner will also be influenced by ambient temperature. Different normalization scans may then be needed at different room temperatures. Scanner room temperature may vary slightly during the day. Hence, it may be necessary to perform normalization scans more than once daily, which is very impractical. However, this is only necessary if the influence of temperature on detector efficiency leads to significant changes in the reconstructed PET images.

We have investigated the temperature dependence of the LabPET APD-based small-animal PET scanner. From these investigations, we aim to derive the temperature stability requirements of the scanner room. First some theoretical considerations about temperature dependence of APD gain and consequently APD-based scanner sensitivity are discussed. Then we have used simulations to estimate the effect of temperature on APD-based scanner sensitivity for different APD temperature coefficients. Thirdly, a number of experiments were performed to evaluate the temperature dependence of the LabPET scanner in our lab. 


\section{MATERIALS AND METHODS}

\section{A. Theory}

1) Temperature dependence of APDs: APDs are photodiodes which possess an internal gain process due to avalanche multiplication. The gain factor $M$ is mainly influenced by bias voltage and temperature. As temperature increases, there are more lattice vibrations and hence a higher probability of electron-phonon interactions. This leads to a higher electron loss, yielding a lower gain[3]. The variation of gain with temperature is defined as:

$$
\tau=\frac{1}{M} \frac{d M}{d T}
$$

and is usually of the order $-2 \%$ to $-4 \% /{ }^{\circ} \mathrm{C}$. This means that, to maintain gain variations below $1 \%$, temperature should be constant within $0.25-0.5{ }^{\circ} \mathrm{C}$.

2) Scanner sensitivity: When a gamma photon is absorbed by a scintillation crystal, the scintillation light produced by the crystal is proportional to the energy of the gamma photon. When this light is detected by the APD, the current output of the APD is also proportional to this energy. Therefore, the energy of an absorbed photon can be determined from the current output of the APD. As the different APDs in a PET scanner may exhibit a slightly different gain even with constant temperature and bias voltage, the current corresponding to a photon of $511 \mathrm{keV}$ is calibrated for each APD.

In PET imaging, an energy window is set to eliminate scattered photons, which have an energy lower than $511 \mathrm{keV}$. This energy window is set on the energy histogram, which depends on correct calibration of the APD-based detector for each crystal, as described above. If the temperature rises, APD gain is reduced and a lower current will be produced by the scintillation light obtained from absorption of a 511 $\mathrm{keV}$ photon. If the APD calibration is not adjusted for this, a fraction of these photons which previously were inside the energy window will now be below the lower energy threshold. Therefore, the sensitivity of the scanner is reduced.

\section{B. Simulations}

It is clear from the explanation above that the relation between APD gain and scanner sensitivity is not a simple linear one. This is due to the shape of the energy histogram. It is therefore difficult to estimate what the effect of an increase of ambient temperature with $1^{\circ}$ will be on scanner sensitivity. We have tried to estimate this using simple MATLAB simulations for different temperatures and different APD temperature coefficients.

The different steps used in this simulation are illustrated in fig. 1. First, a GATE model of a small animal PET scanner with comparable geometry as the $\mathrm{LabPET}^{\mathrm{TM}}$ scanner was used to obtain a simulated energy spectrum for LYSO crystals coupled to APD detectors[4], [5]. A ${ }^{22} \mathrm{Na}$ point source was used in the simulation. The energy resolution was set to $24 \%$. It was assumed that this energy spectrum was obtained at $21^{\circ}$ and that all detectors were calibrated to this energy spectrum. For
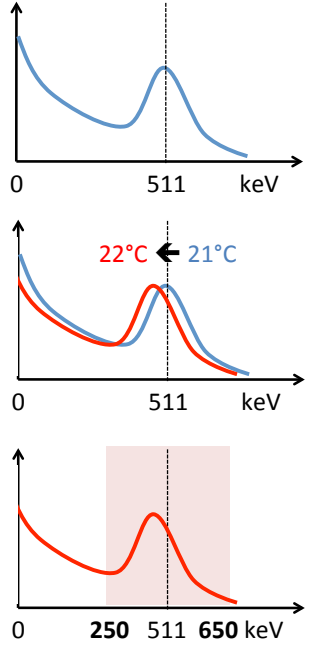

Figure 1. Illustration of the different steps used in the simulation of the effect of temperature-dependent APD changes on PET scanner sensitivity. First a reference energy histogram is simulated at $21^{\circ} \mathrm{C}$. For each temperature, the energy histogram is then shifted left or right over a distance dependent on the temperature difference and the simulated temperature coefficient of the APDs. Finally, the integral is calculated over the original energy window, which was not shifted.

a given APD temperature coefficient and a given temperature shift, the energy spectrum was shifted to left or right by:

$$
\Delta E=511 \tau \Delta T
$$

where $\tau$ is defined above and usually negative, and $\Delta T$ is the difference between the simulated temperature and $21^{\circ} \mathrm{C}$. If $\Delta E$ is positive, the curve is shifted to the right, otherwise to the left. Then, relative scanner sensitivity is obtained by calculating the integral of the shifted energy spectrum between the thresholds set by the original energy window (250 $650 \mathrm{keV}$ ) and dividing by the integral at $21^{\circ} \mathrm{C}$. This was done for temperatures ranging from $18^{\circ} \mathrm{C}$ to $26^{\circ} \mathrm{C}$ and $\mathrm{APD}$ temperature coefficients of $-1 \%$ to $-4 \%$.

\section{Experiments}

After theoretical simulations, experimental evaluation of the temperature dependence of the $\mathrm{LabPET}^{\mathrm{TM}}{ }_{\text {scanner was also }}$ performed. Five experiments were done. First, the influence of short term (minutes) temperature variations on scanner sensitivity is evaluated. Second, the effect of acquiring at different temperatures which have stabilized for a day (long term, days) is investigated. Then the influence of temperature on the axial sensitivity profile was measured. The effect on absolute quantification was also investigated. Finally long-term stability under stable temperature was evaluated.

1) Hardware: All acquisitions were done on a Triumph II system (Gamma Medica - Ideas). This tri-modality system consists of a micro-PET $\left(\mathrm{LabPET}^{\mathrm{TM}}\right)$, micro-CT $\left(\mathrm{X}-\mathrm{O}^{\mathrm{TM}}\right)$ and micro-SPECT (X-SPECT ${ }^{\circledR}$ ) scanner. The LabPET $^{\mathrm{TM}}{ }_{\text {scanner }}$ is an APD-based scanner with $50 \%$ LYSO and $50 \%$ LGSO 
crystals $(2 \times 2 \times 12.6 \mathrm{~mm})[6]$, [7]. The $\mathrm{X}-\mathrm{O}^{\mathrm{TM}}$ is a microCT scanner with adjustable magnification. The X-SPECT ${ }^{\circledR}$ scanner was not used in this work.

In all experiments room temperature was monitored using a USB temperature logger (THL2 Temperature / Humidity USB Datalogger, UEI Test Instruments, Beaverton, USA). Samples were taken every minute. The temperature logger was always placed in the same location in the center of the scanner room, at about $1 \mathrm{~m}$ from the bore of the scanner.

2) Short term temperature variation: To test the immediate effect of temperature changes on scanner sensitivity, a calibrated ${ }^{22} \mathrm{Na}$ point source was imaged while varying room temperature. The point source, which contained $200 \mathrm{kBq}$ at the time of acquisition, was placed in the center of the fieldof-view (FOV). Temperature was varied between $21^{\circ} \mathrm{C}$ and $28^{\circ} \mathrm{C}$. The total acquisition duration was 4.5 hours. Room temperature was varied by changing airconditioner settings.

After acquisition, the PET data was grouped into frames of 10 minutes. Within each frame, the total number of counts was calculated. The scanner sensitivity (in $\mathrm{cps} / \mathrm{Bq}$ ) within each frame was calculated as:

$$
S_{i}=\frac{N_{i}}{A_{\text {ref }} \Delta t}
$$

where $N_{i}$ is the total number of counts in frame $i, A_{\text {ref }}$ is the activity in the ${ }^{22} \mathrm{Na}$ point source at the time of acquisition (in $\mathrm{Bq}$ ) and $\Delta t$ is the frame duration (in seconds). The average temperature was also calculated for each frame. Sensitivity and temperature versus time were plotted and linear regression was used to determine the relationship between temperature and sensitivity.

3) Long term temperature variation: It is possible that short term changes do not affect scanner sensitivity immediately, or that scanner sensitivity is only transiently changed with temperature changes and normalizes again after a period of time. To evaluate the effect of sustained and stable temperature changes, measurements of the same ${ }^{22} \mathrm{Na}$ point source were performed on subsequent days at different temperatures between 21 and $25^{\circ} \mathrm{C}$ which had stabilized for at least 6 hours. The point source was placed in the center of the FOV and the acquisition time was $5 \mathrm{~min}$. A total of 15 data points were acquired. Scanner sensitivity was calculated for each acquisition using the equation described above. Linear regression was performed on the data points to determine the relationship between temperature and sensitivity.

4) NEMA axial sensitivity profile: The NEMA NU 4 - 2008 standard for performance measurements of small animal PET scanners requires acquisition of the sensitivity profile along the axial direction in the transaxial center of the scanner. This was done by placing the calibrated ${ }^{2} 2 \mathrm{Na}$ point source in the (transaxial and axial) center of the FOV and moving the point source in $2 \mathrm{~mm}$ steps from the center outwards in both axial directions. At each position, an acquisition of 1 min was performed. A blank scan was also performed to estimate background counts. The sensitivity in each position

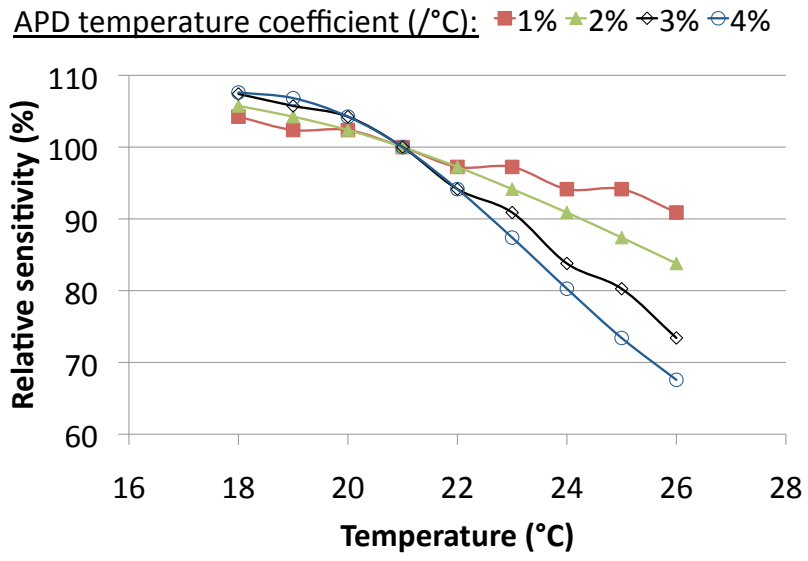

Figure 2. Simulated scanner sensitivity for different temperatures and different APD temperature coefficients, relative to a reference temperature of $21^{\circ} \mathrm{C}$.

was calculated using the NEMA methodology. First, single slice rebinning is used to collapse $3 \mathrm{D}$ sinograms into $2 \mathrm{D}$ sinograms. Then, for each row (angle) of the sinogram, the bin with the highest number of counts is determined and this bin is aligned with the center of the row. Then, all counts located more than $1 \mathrm{~cm}$ from the center of each row are set to zero. The same processing is done on the sinogram obtained from the blank scan. The sensitivity at each axial position is then calculated as:

$$
S_{N E M A, i}=\frac{N_{N E M A, i}-N_{N E M A, B, i}}{A_{\text {ref }} \Delta t}
$$

where $N_{N E M A, i}$ and $N_{N E M A, B}$ represent the total counts in the processed sinograms of axial step $i$ and the blank scan respectively.

5) Quantification: To determine the effect of temperature changes on quantitative accuracy of reconstructed PET images, a quantification study with the NEMA image quality phantom was performed. PET images were acquired on two days. On the first day, the room temperature was kept at $21^{\circ} \mathrm{C}$. On the second day the room temperature was set to $23{ }^{\circ} \mathrm{C}$. On each day a normalization scan was also acquired at the same room temperature. The phantom was filled with approximately 18 $\mathrm{MBq}$ for both acquisitions. A calibrated ${ }^{22} \mathrm{Na}$ point source was also placed in the FOV to allow calculation of the absolute activity concentration using our previously described method[8]. In that method, the reconstructed activity in the point source is used to calculate a calibration factor for absolute activity derivation.

PET images were reconstructed with listmode ML-EM reconstruction using CT-based attenuation correction and single scatter simulation (SSS) based scatter correction. Both PET data sets were reconstructed twice: once using the correct normalization scan and once using the normalization scan acquired at a different temperature. In each of the four reconstructed images, the absolute activity concentration in the uniform region of the phantom was calculated. The calculated activity concentration was compared to the activity 


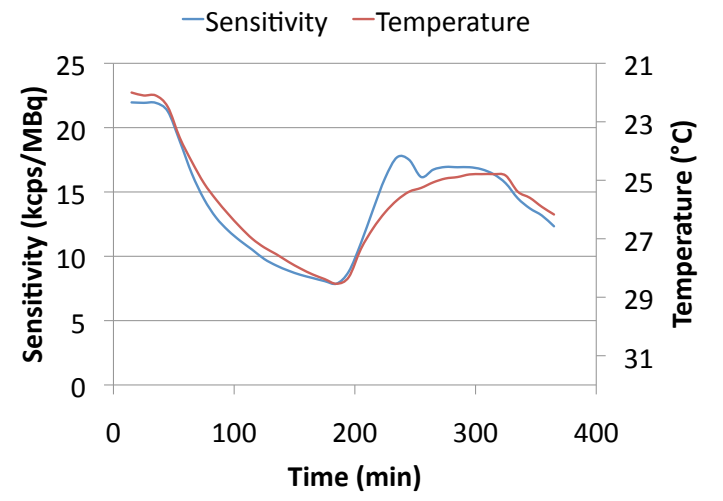

Figure 3. Variation of sensitivity and room temperature over time during a point source acquisition with varying airconditioner settings. It should be noted that the temperature scale is inverted and shown on the right-hand side.

concentration obtained from dose calibrator and volume measurements.

6) Day-to-day variation: Demonstrating the influence of temperature on scanner performance does not imply that scanner stability can be ensured if the ambient temperature is kept constant. It is also necessary to evaluate the feasibility of obtaining a constant room temperature. The variability of scanner performance with constant temperature should also be investigated. This was done by performing normalization scans using a rotating ${ }^{68} \mathrm{Ge}$ rod source daily during a period of 4 days. The room temperature was kept as stable as possible around $20{ }^{\circ} \mathrm{C}$ and was monitored using the USB temperature logger. The total number of counts and the average temperature were calculated for each normalization scan. The standard deviation of both were calculated as a measure of variation.

\section{RESULTS}

\section{A. Simulations}

Fig. 2 shows simulated scanner sensitivity at different temperatures for different APD temperature coefficients. Sensitivity is calculated relative to the sensitivity at $21^{\circ} \mathrm{C}$. The relative change is much smaller when temperature is decreased relative to the reference temperature than when it is increased. E.g. with a temperature coefficient of $2 \%$, a relative sensitivity decrease of $10 \%$ is seen if room temperature increases with $3^{\circ} \mathrm{C}$, whereas an increase of only $5 \%$ is observed when room temperature is decreased with $3^{\circ} \mathrm{C}$.

\section{B. Experiments}

1) Short term temperature variation: The immediate effect of room temperature variations on scanner sensitivity is depicted in fig. 3. Linear regression showed a change of sensitivity of $-2.25 \mathrm{kcps} / \mathrm{MBq}$ per ${ }^{\circ} \mathrm{C}$. The correlation coefficient of temperature and sensitivity was -0.96 .

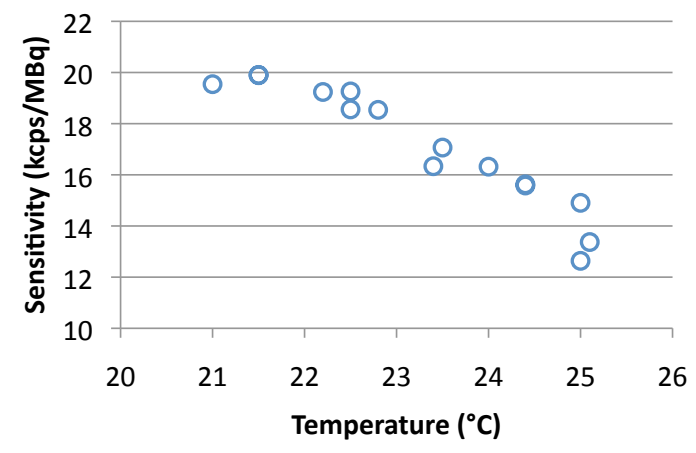

Figure 4. Scanner sensitivity at different stable temperatures.

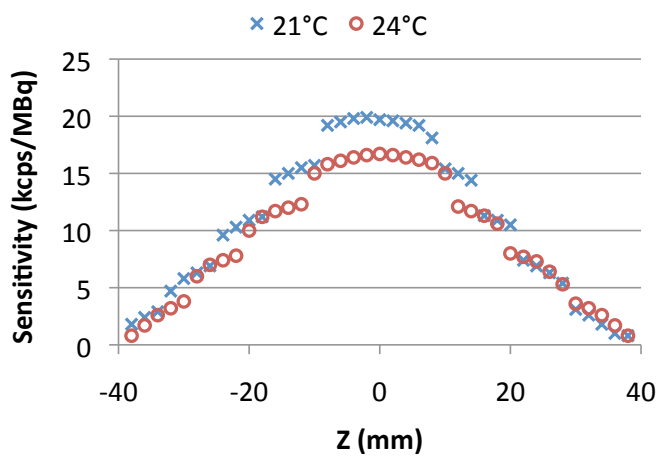

Figure 5. Axial sensitivity profile at two different temperatures measured using the NEMA NU 4 - 2008 standard.

2) Long term temperature variation: The effect of sustained and stable temperature changes is shown in fig. 4. A change of sensitivity of $-1.64 \mathrm{kcps} / \mathrm{MBq}$ per ${ }^{\circ} \mathrm{C}$ is derived using linear regression. A strong correlation between temperature and sensitivity is again observed, with a correlation coefficient of 0.95 .

3) NEMA axial sensitivity profile: The peak sensitivity measured with the NEMA sensitivity protocol changes from $19.9 \mathrm{kcps} / \mathrm{MBq}$ to $16.6 \mathrm{kcps} / \mathrm{MBq}$ when ambient temperature is increased from $21^{\circ} \mathrm{C}$ to $24^{\circ} \mathrm{C}$, corresponding to a change of peak sensitivity of $-1.1 \mathrm{kcps} / \mathrm{MBq}$ per ${ }^{\circ} \mathrm{C}$. The sensitivity at $1 / 4$ of the axial FOV of the scanner is also decreased from $10.5 \mathrm{kcps} / \mathrm{MBq}$ to $8 \mathrm{kcps} / \mathrm{MBq}$. The axial sensitivity profile at both temperatures is shown in fig. 5 .

4) Quantification: Fig. 6 shows transverse slices through both images reconstructed from the acquisition of the image quality phantom at $23^{\circ} \mathrm{C}$. Fig. 7 depicts the results of the calculation of the absolute activity concentration from all 4 images. It shows that reconstructing with correct normalization (acquired at the same room temperature) leads to a quantification accuracy of $97 \%$ and $99 \%$ at $21^{\circ} \mathrm{C}$ and $23{ }^{\circ} \mathrm{C}$ respectively. Reconstructing with incorrect normalization leads to less accurate quantification: $96 \%$ and $87 \%$ respectively. 

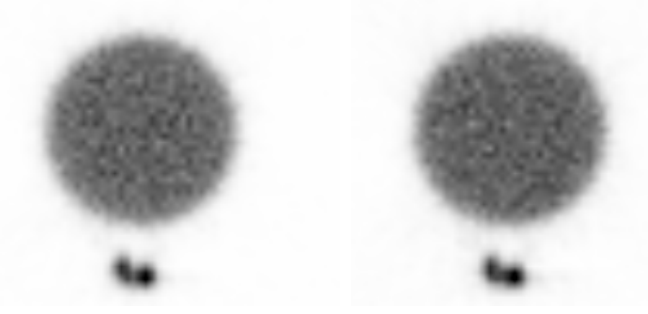

Figure 6. Transverse slice through the uniform section of the Image Quality phantom, acquired at room temperature of $23^{\circ} \mathrm{C}$ and reconstructed using a normalization scan acquired at $23^{\circ} \mathrm{C}$ (left) and $21^{\circ} \mathrm{C}$ (right). The reference $\mathrm{Na}-22$ point source is also visible at the bottom.

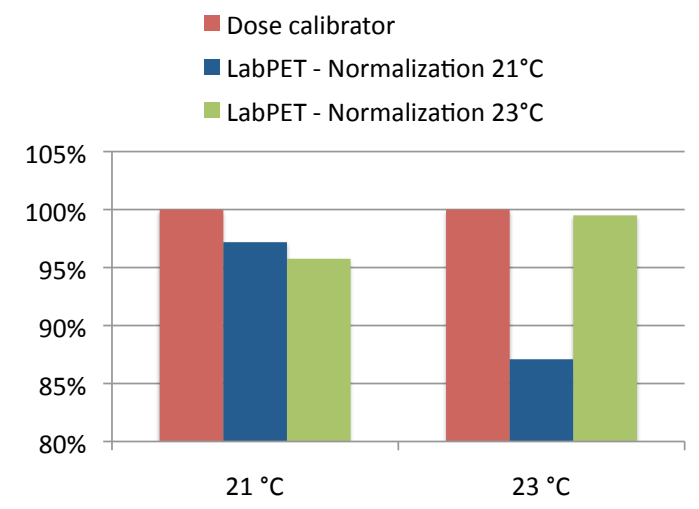

Figure 7. Accuracy of calculation of absolute activity concentration from the Image Quality phantom acquisition. The acquisitions at $21^{\circ} \mathrm{C}$ and $23^{\circ} \mathrm{C}$ were reconstructed using normalization scans acquired at both temperatures. The activity concentrations are normalized to the activity concentration measured with the dose calibrator.

5) Day-to-day variation: Fig. 8 shows the total counts and average temperature for normalization scans acquired during 4 consecutive days. The relative standard deviations of temperature and counts were $0.19^{\circ} \mathrm{C}$ and 10.2 Mcts (1.33 $\%)$ respectively.

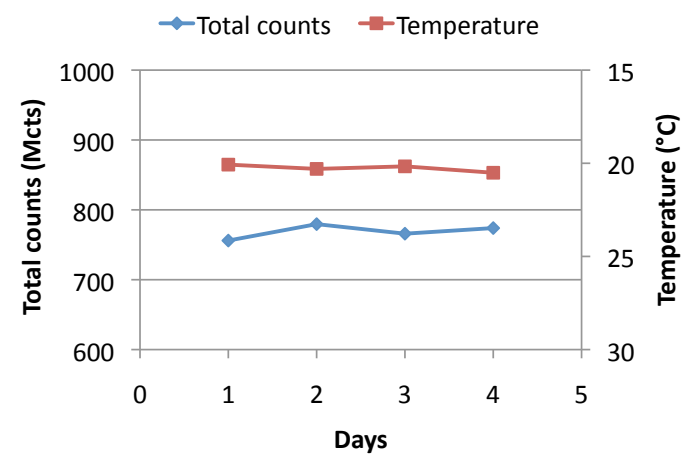

Figure 8. Total counts in normalization scans performed on 4 consecutive days and average temperatures during these normalization scans.

\section{DISCUSSION}

We have first estimated the effect of different APD temperature coefficients on the variation of scanner sensitivity with temperature. As was to be expected, a higher APD temperature coefficient leads to larger variations in scanner sensitivity. It was also conceivable that a larger temperature shift from the reference temperature (at which the APDbased detector was calibrated) leads to a larger deviation of sensitivity. However, a change of APD gain with $1 \%$ does not lead to a corresponding change in relative sensitivity of $1 \%$, but rather a much higher change. This is due to the complex non-uniform shape of the energy histogram. It should also be noted that the relationship between scanner sensitivity and room temperature is not simply linear over the entire range. Smaller relative changes of sensitivity are observed when room temperature is decreased from the reference temperature than when it is increased. This can also be related to the shape of the energy histogram.

As we expected from the simulations, a large variation of scanner sensitivity was observed with varying temperature. When room temperature is changed, this immediately affects scanner sensitivity, causing a drop of sensitivity by -2.25 $\mathrm{kcps} / \mathrm{MBq}$ for each ${ }^{\circ} \mathrm{C}$ the temperature is increased. As the sensitivity of the LabPET scanner is between 10 and 25 $\mathrm{kcps} / \mathrm{MBq}$, this means that scanner sensitivity immediately changes $10 \%$ or more per ${ }^{\circ} \mathrm{C}$. This may lead to errors in dynamic PET scans: if temperature changes between different frames of a dynamic scan, frames will be acquired at a different sensitivity. Hence, the images reconstructed from these frames can not be compared quantitatively.

Even if scanner sensitivity is kept constant during the total acquisition time, room temperature may change over days. Our evaluation shows that the effect of stabilized room temperature changes is smaller $\left(-1.64 \mathrm{kcps} / \mathrm{MBq}\right.$ per $\left.{ }^{\circ} \mathrm{C}\right)$ than the immediate effect of room temperature changes, but is still important. It is unclear why this effect is smaller. Perhaps this is due to the integrated cooling system of the scanner, which partially compensates room temperature increases. As the system is air-cooled, it is possible that the cooling system can not compensate the full increase in room temperature. The NEMA axial sensitivity profile measurements also show an even smaller influence $\left(-1.1 \mathrm{kcps} / \mathrm{MBq}\right.$ per $\left.{ }^{\circ} \mathrm{C}\right)$, which is nevertheless still a decrease in sensitivity of $5 \%$ per ${ }^{\circ} \mathrm{C}$.

The results of the experiments with the image quality phantom show that using a normalization scan acquired at the same room temperature as the acquisition is necessary. Especially for the acquisition at $23^{\circ} \mathrm{C}$, a large quantification error is observed if the normalization scan at $21^{\circ} \mathrm{C}$ is used. A smaller error is seen when the acquisition at $21^{\circ} \mathrm{C}$ is reconstructed using the normalization scan acquired at $23^{\circ} \mathrm{C}$. This difference can be due to the fact that both scans were acquired on different days, and the phantom was therefore not exactly in the same position on both days. Therefore, differences in the normalization scans may have had a significant effect on one acquisition and not on the other.

In the last experiment we have demonstrated that it is possible to keep scanner sensitivity relatively constant when room 
temperature is kept constant. This means that a normalization scan does not need to be performed every day (or more), assuming that room temperature can be kept constant. In that same experiment it was shown that it is possible to keep room temperature sufficiently constant.

\section{CONCLUSION}

We have investigated the temperature dependence of the LabPET small-animal PET scanner. We have shown that the sensitivity of the scanner strongly depends on room temperature. A larger effect is observed in the period directly after a temperature change than after the temperature change has stabilized. For accurate quantification, temperature-dependent detector efficiency normalization should be used. However, if the room temperature is kept constant, good scanner stability is observed. Based on these results we conclude that if scanner room temperature is kept constant within $0.5^{\circ} \mathrm{C}$, weekly normalization scans should be sufficient.

\section{ACKNOWLEDGEMENTS}

This work was supported by the EU FP7 project SUBLIMA (Grant Agreement $\mathrm{Nr}$ 241711). Christian Vanhove is supported by

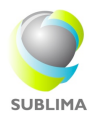

the GROUP-ID consortium from Ghent University. Roel Van Holen is supported by a postdoctoral fellowship of the Research Foundation Flanders (FWO).

\section{REFERENCES}

[1] R D Badawi, M A Lodge, and P K Marsden, "Algorithms for calculating detector efficiency normalization coefficients for true coincidences in $3 \mathrm{~d}$ pet," Physics in Medicine and Biology, vol. 43, no. 1, pp. 189-205, Jan 1998.

[2] G Delso and S Ziegler, "Pet/mri system design," Eur J Nucl Med Mol Imaging, vol. 36 Suppl 1, pp. S86-92, Mar 2009.

[3] JP Pansart, "Avalanche photodiodes for particle detection," Nucl Instrum Meth A, vol. 387, pp. 186-93, Jan 1997.

[4] S Jan, G Santin, D Strul, S Staelens, K Assie, D Autret, S Avner, R Barbier, M Bardies, P Bloomfield, D Brasse, V Breton, P Bruyndonckx, I Buvat, A Chatziioannou, Y Choi, Y Chung, C Comtat, D Donnarieix, L Ferrer, S Glick, C Groiselle, D Guez, P Honore, S Kerhoas-Cavata, A Kirov, V Kohli, M Koole, M Krieguer, D van der Laan, F Lamare, G Largeron, C Lartizien, D Lazaro, M Maas, L Maigne, F Mayet, F Melot, C Merheb, E Penacchio, J Perez, U Pietrzyk, F Rannou, M Rey, D Schaart, C Schmidtlein, L Simon, T Song, J Vieira, D Visvikis, R Van de Walle, E Wieers, and C Morel, "Gate: a simulation toolkit for pet and spect," Phys Med Biol, vol. 49, pp. 4543-61, Jan 2004.

[5] S Rechka, R Fontaine, M Rafecas, and R Lecomte, "Development and validation of a gate simulation model for the labpet scanner," IEEE T Nucl Sci, vol. 56, pp. 3672-9, Jan 2009.

[6] M Tetrault, N Viscogliosi, J Riendeau, F Bélanger, J Michaud, H Semmaoui, P Bérard, F Lemieux, L Arpin, M Bergeron, J Cadorette, CM Pepin, G Robert, M Lepage, R Lecomte, and R Fontaine, "System architecture of the labpet ${ }^{\mathrm{TM}}$ small animal pet scanner," IEEE T Nucl Sci, Jan 2008.

[7] R Fontaine, F Belanger, N Viscogliosi, H Semmaoui, M Tetrault, J Michaud, C Pepin, J Cadorette, and R Lecomte, "The hardware and signal processing architecture of labpet ${ }^{\mathrm{TM}}$, a small animal apd-based digital pet scanner," Nuclear Science, IEEE Transactions on, vol. 56, no. 1, pp. $3-9,2009$.

[8] V Keereman, R Van Holen, C Vanhove, P Mollet, and S Vandenberghe, "Absolute quantification for small-animal pet," IEEE Nucl Sci Conf $R$, pp. 3715-9, 2011. 